Enfoques. Revista de Investigación en Ciencias de la Administración http://doi.org/10.33996/revistaenfoques.v4i16.97 No. 16 | Volumen 4 | Octubre - Diciembre 2020 http://revistaenfoques.org ISSN: $2616-8219$ ISSN-L: $2616-8219$ pp. $248-260$

\section{ESTRATEGIAS PARA EL INCREMENTO DE VENTAS: CASO DE ESTUDIO MICROEMPRESA MUNDO DE ENSUEÑOS}

\author{
STRATEGIES FOR INCREASING SALES: A CASE STUDY MICRO- \\ ENTERPRISE WORLD OF DREAMS
}

\section{ESTRATÉGIAS PARA AUMENTAR AS VENDAS: UM ESTUDO DE CASO MICROEMPRESA MUNDO DE SONHOS}

\author{
Fernando Terán Guerrero \\ y Nery Elisabeth García Paredes
}

Artículo recibido julio 2020 | Arbitrado agosto 2020 | Publicado 01 de octubre 2020

\section{Resumen}

El propósito de esta investigación es presentar un estudio descriptivo y exploratorio que buscó apoyar a la gestión del conocimiento de tal forma de como las empresas están usando las estrategias para incrementar el valor de los clientes y se reflejen en las ventas. El foco de esta investigación es una de las empresas del sector. Los resultados muestran que las estrategias de marketing empleadas por la empresa propuesta presentan una significativa orientación al cliente, sin embargo, se destacan por seguir estrategias de crecimiento y diversificación de marketing en cada uno de los tres autores analizados para aumentar el valor de los clientes. Se concluye que los clientes estaban satisfechos con el producto conseguido al momento de realizar una compra, pero el problema estaba en que no retornaban e inmediatamente de un determinado tiempo no regresaban, como resultado después de la encuesta se decidió fidelizar a los clientes que ya eran leales.

Palabras clave: Estrategias, incremento en ventas, estrategias genéricas, estrategias de crecimiento, cliente

\begin{abstract}
The purpose of this research is to present a descriptive and exploratory study that seeks to support knowledge management in such a way that companies are using strategies to increase customer value and be reflected in sales. The focus of this research is one of the companies in the sector. The results show that the marketing strategies used by the proposed company have a significant customer orientation, however, they stand out for following marketing growth and diversification strategies in each of the three authors analyzed to increase the value of customers. It is concluded that customers were satisfied with the product obtained at the time of making a purchase, but the problem was that they did not return and immediately after a certain time they did not return, as a result after the survey it was decided to retain customers who were already loyal.
\end{abstract}

Key words: Strategies; increase in sales; generic strategies; growth strategies, customer
Fernando Terán Guerrero

nandot28@gmail.com

ORCID: 0000-0002-6454-482X

Universidad Técnica de Ambato, Ecuador.

Docente de Academia Miguel Iturralde, del Instituto Tecnológico Victoria Vásconez Cuvi, de la Universidad Técnica de Cotopaxi, del Instituto Tecnológico La Maná y Docente de la Universidad Técnica de Ambato, Ecuador.

\section{Nery Elisabeth García Paredes}

sarbagflorecuador2015@gmail.com ORCID: 0000-0001-5267-5868

Universidad Técnica de Ambato, Ecuador.

Magister en Matemática y Educación Superior. Dra. Física y Lcda. En Física y Matemática. Experiencia: Docente de Matemática Superior y Física Superior en Educación media por 25 años; Docente de Ciencias Exactas Bachillerato Internacional; Docente Universitario por 2 años en las asignaturas de Física y Cálculo diferencial e Integral, Gerente General administrativo y de Relaciones exteriores por 10 años. Universidad Técnica De Ambato, Ecuador.

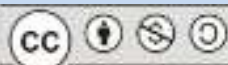




\section{Resumo}

O objetivo desta pesquisa é apresentar um estudo descritivo e exploratório que visa apoiar a gestão do conhecimento de forma a como as empresas estão utilizando estratégias para aumentar o valor dos clientes e se refletem nas vendas. O foco desta pesquisa é uma das empresas do setor. Os resultados mostram que as estratégias de marketing utilizadas pela empresa proposta apresentam uma orientação significativa para o cliente, porém, destacam-se por seguir estratégias de crescimento e diversificação de marketing em cada um dos três autores analisados para aumentar o valor dos clientes. Conclui-se que os clientes ficaram satisfeitos com o produto obtido no momento da realização da compra, mas o problema é que eles não devolveram e imediatamente após um determinado tempo não devolveram, por isso, após a pesquisa, decidiu-se reter os clientes que já estavam fiel.

Palavras-chave: Estratégias; aumento de vendas; estratégias genéricas; estratégias de crescimento, cliente

\section{INTRODUCCIÓN}

El mundo globalizado y cambiante en el que se desarrollan las empresas en la actualidad, existen múltiples obstáculos que no permiten un adecuado direccionamiento hacia los objetivos que se plantean las organizaciones, sin embargo, es tarea de los expertos del mercado planear estrategias que les permitan alcanzar un crecimiento sostenible a través del tiempo. Los mercados cada vez son más exigentes, el éxito de una organización está dado en gran medida por la capacidad que tienen las empresas para adaptarse a las nuevas y versátiles tendencias que se dan en su entorno, es decir establecer las necesidades de los consumidores.

Tenazmente los investigadores han desarrollado cuáles compensarían ser las mejores estrategias de marketing, que compensan a realizar las empresas (Farías
Nazel, 2014; Chou y Chen, 2004; Hogan, Lemon y Libai, 2004). Una vez que los esfuerzos de marketing se encuentran correctamente alineados con las necesidades del mercado, se podrá obtener resultados favorables para la organización que en la mayoría de los casos se traduce como la generación de rentabilidad por la inversión realizada.

Los negocios nuevos enfrentan múltiples desafíos en su creación y la consolidación de los mismos, un factor determinante son los altos costos de los arriendos en las zonas comerciales, lo que les quita eficiencia y competitividad, debido a arriendos costosos, los precios de los artículos son altos. Las ventas en los primeros años no son tan elevadas por lo que muchos negocios no pueden pagar los arriendos y se ven forzados a cerrar.

Naim (2002), manifiesta que en etapas económicos en los que la competencia es cada vez más intensa, es preciso utilizar correctamente las diferentes herramientas que el marketing, brinda para alcanzar fortalecimiento y estabilidad en el mercado, en la cual la gestión estratégica de ventas es crucial y terminante para la competitividad de la empresa. Todo negocio opera en forma de un ciclo o un círculo, donde se compra productos, se vende y se gana dinero: El comprar, vender y ganar dinero se repite constantemente, la empresa empieza a crecer alrededor de este ciclo, volviéndose un negocio rentable. El crecimiento es uno de los objetivos que se ha planteado el dueño del negocio. Su propietario ha querido que el negocio progrese en forma planificada y organiza.

Lamentablemente, dicho crecimiento se ha estancado. Desde su apertura este bazar ha ido teniendo un crecimiento anual, pero desde hace tres años se ha ido observando 
que hay un estancamiento en las ventas. A esta conclusión se ha podido llegar por los registros de venta que se llevan mensualmente en este negocio, y este ha sido el mecanismo que permitido comparar mes a mes y año a año. Este estancamiento en las ventas ha originado una liquidez modesta y esto refleja que el negocio no pueda seguir creciendo, y no pueda diversificar sus utilidades ni aumentar sus márgenes de ganancia.

En el almacén una de las debilidades que se ha podido detectar es que nunca se ha utilizado promociones, descuentos, ofertas y regalos de suvenir en fechas especiales como Navidad, San Valentín, el Día de la Madre por el desconocimiento que se tiene para la aplicación de dichas técnicas de mercadeo.

El presente trabajo de investigación tiene la finalidad de detectar y analizar cuáles son los factores que están influyendo para que haya un estancamiento en las ventas por tres años consecutivos. Con los resultados de esta investigación se podría tener mecanismos acertados y no empíricos para un crecimiento prolongado del negocio. Con este trabajo se busca aplicar nuevas formas de administrar y de hacer negocios lo que originaría una innovación al manejo de este bazar de una forma más técnica para una consolidación en el mercado y crear fortalezas y oportunidades en un mundo tan cambiante y variable como el actual.

\section{MÉTODO}

El nivel de investigación que se aplicó en este estudio fue de carácter descriptivo y exploratorio. Al respecto Sampieri (2003), enuncia que "las investigaciones de tipo descriptivas buscan especificar propiedades, características y rasgos importantes de cualquier fenómeno que se analice" (p.119)
Se utilizó el método descriptivo, porque alcanzó el registro, análisis e interpretación de la naturaleza de los fenómenos con el objetivo de obtener conclusiones sobre la conducta, gustos y predilecciones de las personas que visitan este local (Tamayo, 2004).

La población se tomó en cuenta el PEA de Latacunga que el $54,5 \%$ de la población de 170500 habitantes; por lo que existen 92923 personas como universo (INEC, 2010), proyección para 2019. Para la investigación de mercados se utilizó el muestreo probabilístico aleatorio simple.

Se realizó una prueba piloto a diez personas y esta encuesta piloto permitió determinar las probabilidades de éxito y fracaso ( $p$ y $q$ ), mediante la pregunta filtro (¿Conoce usted los productos que ofrece el almacén "MUNDO DE ENSUEÑOS"?). Luego de la información recabada se determinó que: $p=0,8$ y $q=0,2$

De acuerdo con Fischer y Navarro (1996), para poblaciones finitas (menos de 500 mil elementos), que fue el caso aquí, se utiliza la fórmula:

$$
n=\frac{\sigma^{2} N p q}{e^{2}(N-1)+\sigma^{2} p q}
$$

Donde:

$\mathrm{n}=$ muestra

$\mathrm{N}=$ población $(92923)$

$\mathrm{p}=$ probabilidad de ocurrencia del suceso (0.8)

$q=$ probabilidad de no ocurrencia del suceso (0.2)

$Z$ = nivel de confianza $(95 \%=1.96)$ datos basados en las tablas estadísticas $e=5 \%$ 


$$
n=\frac{1,96^{2} \times 0,8 \times 0,2 \times 92923}{0,05^{2} x(92923-1)+1,96^{2} x 0,8 \times 0,2}
$$

$n=245$ encuestados

Una vez aplicada la encuesta se procedió a la realización de la tabulación y codificación de cada una de las preguntas mediante la utilización del programa SPSS, mediante la opción de gráficos, los cuales fueron analizados e interpretados por el investigador.

\section{Análisis e interpretación}

Se pudo observar que el mayor porcentaje de personas encuestadas, es decir el $34 \%$ se encuentra entre los 29 y 35 años de edad, $28 \%$ se encuentra entre los 22 y 28 años de edad, mientras que el grupo que tuvo menor representación fue el integrado por personas mayores de 43 años con un $11 \%$.
En relación al género se apreció que el $71 \%$ de la población correspondía al masculino, mientras que el $29 \%$ corresponde al femenino. En cuento a la demografía o sector de la ciudad de procedencia del encuestado al momento de realizar la compra, se determinó que el $50 \%$ proviene del sector norte de la ciudad; por otro lado, el $23 \%$ proviene del sector centro y el $21 \%$ del sur de la ciudad de Latacunga.

\section{RESULTADOS}

\section{Ocasiones de compras}

Se puede apreciar que de la población encuestada más de un veinte por ciento (27\%) adquiere un peluche en el día de San Valentín; es importante mencionar que el $26 \%$ adquiere el producto en Navidad, lo que significa que para las personas encuestadas estas dos fechas son las más importantes en el año. (Gráfico 1)

1. ¿En qué ocasiones usted compra un peluche?
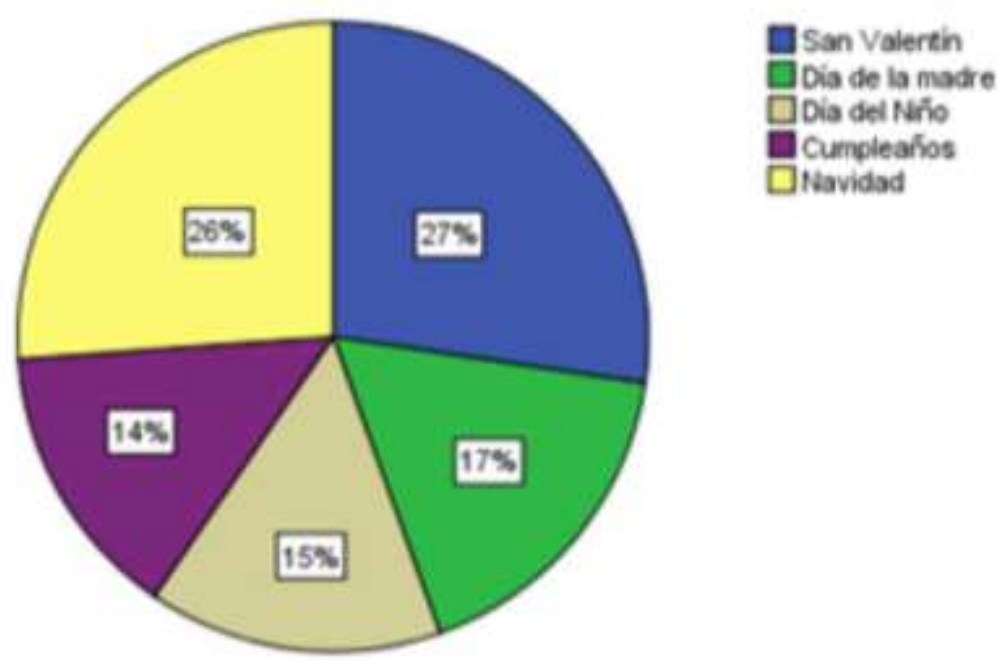

Gráfico 1. Ocasiones usted compra 


\section{Decisión de compra}

En el gráfico 2 se puede observar que en mayor proporción (29\%) los encuestados consideraron la calidad como el factor más importante para realizar la compra, seguido del precio con un $23 \%$, y el tamaño con un $19 \%$; se puede observar que para los encuestados el factor figura fue relevante con un $14 \%$.

\section{De las opciones que se presentan a continuación,} ¿califique por qué compra el producto?

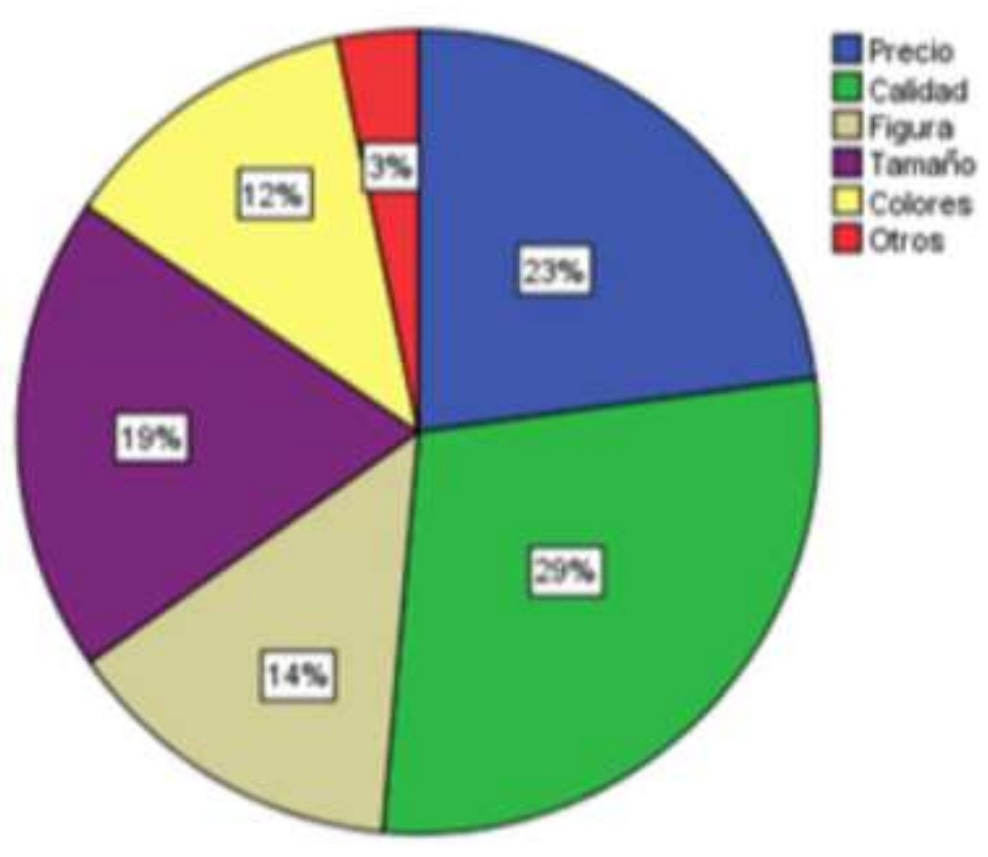

Gráfico 2. Decisión de compra

\section{Cuánto gasta en comprar}

De acuerdo a la información recogida se observa que el mayor porcentaje de personas consultadas con un $43 \%$, asigna un presupuesto de $\$ 5,1$ a $\$ 10$ para la compra de peluches; personas consultadas con un $24 \%$, asigna un presupuesto de $\$ 10,1$ a $\$ 15$, por otro lado, el $23 \%$ asigna un presupuesto mayor a \$15. (Gráfico 3) 
3. ¿Cuando compra peluches, cuánto gasta?

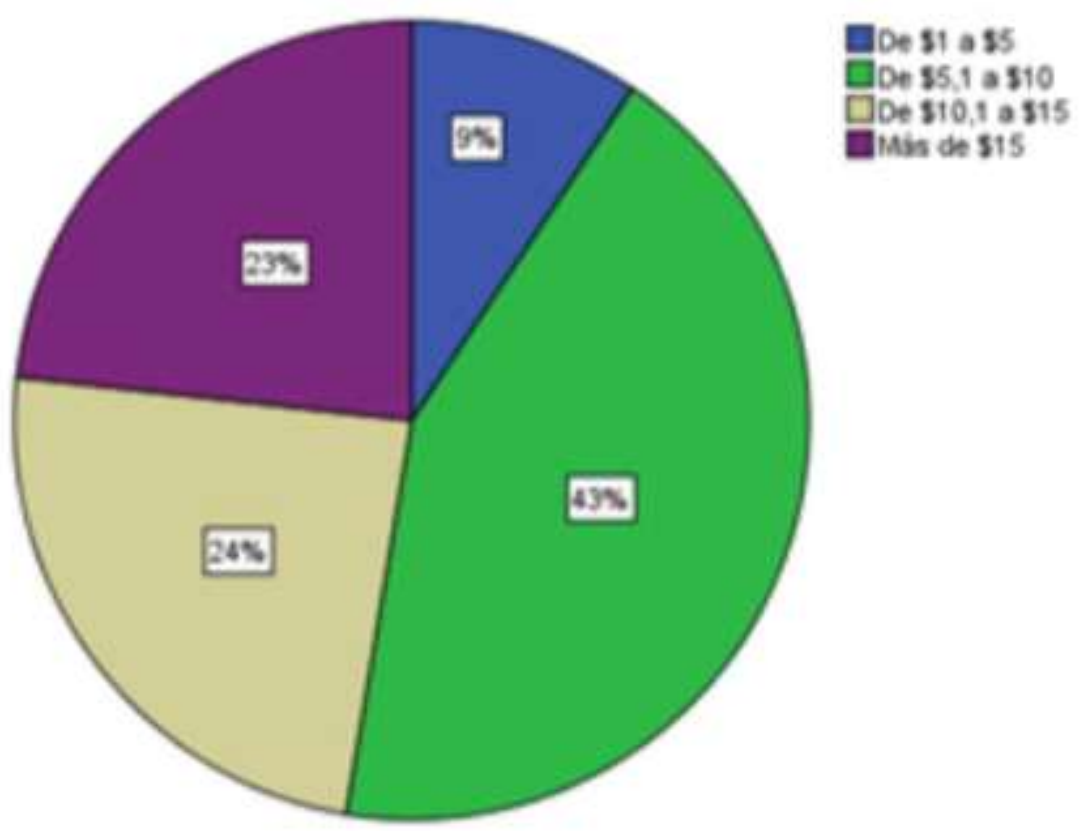

Gráfico 3. Cuánto gasta en comprar

\section{Lugar de preferencia en comprar}

De acuerdo a la información recogida se estimaron que más del treinta por ciento (36\%) de la población encuestada adquiere los peluches en centros comerciales; un $31 \%$ lo hacen en un bazar; por otro lado, solo el $19 \%$ compra el producto en jugueterías y el $14 \%$ en ferias.

\section{Adquirir en "MUNDO DE ENSUEÑOS"}

De acuerdo a los datos obtenidos en la investigación de mercados se pudo determinar que más del cincuenta por ciento afirmaron no conocen los productos que ofrece el negocio de peluches "MUNDO DE ENSUEÑOS".

\section{Precios según la competencia}

De acuerdo a los datos obtenidos en la pregunta diez se pudieron determinar que más del cuarenta por ciento (44\%) de la población encuestada (90) considera que los precios son bajos, (42\%) de la población encuestada considera que los precios son normales y solo un $13 \%$ opina que los precios son altos. (Gráfico 4) 
4. ¿Considera usted que los precios de los productos, en relación a la competencia son?
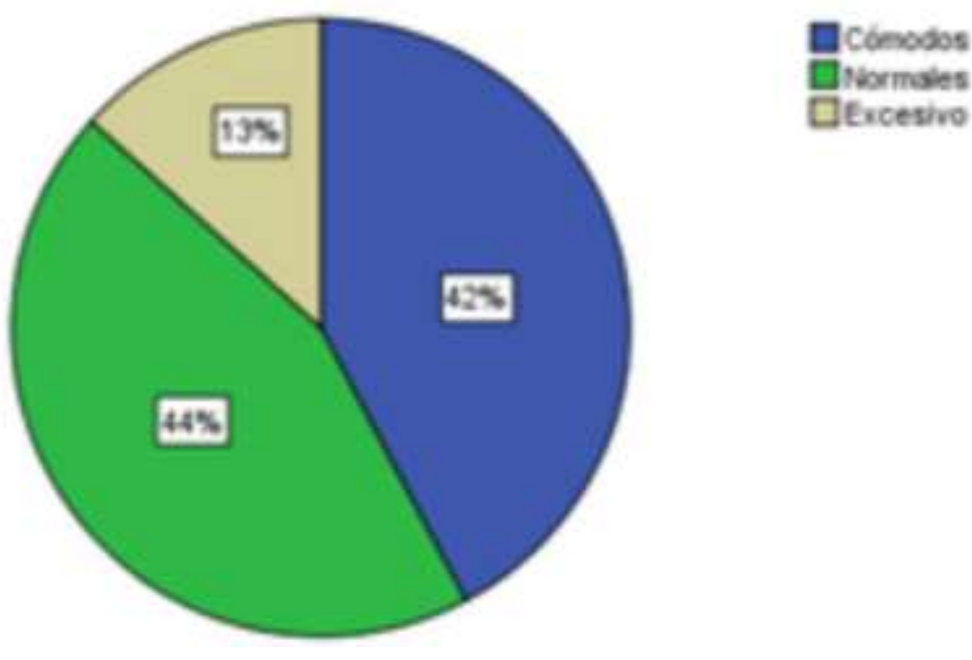

Gráfico 4. Precios según la competencia

\section{Otros productos}

Del total de personas encuestadas el $42 \%$ considera que el negocio debería incluir en su línea de productos tarjetas; por otro lado, más del $50 \%$ corresponde a piñatas y chocolates dentro de la línea de los peluches

\section{Análisis de aceptación a través de los medios de comunicación}

De acuerdo a los datos obtenidos el $36 \%$ dijo que sería mejor conocer el negocio a través de la Radio, seguido del $30 \%$ de volantes e Internet con el $24 \%$.

5. ¿Por qué medio de comunicación le gustaría Conocer nuestros productos?

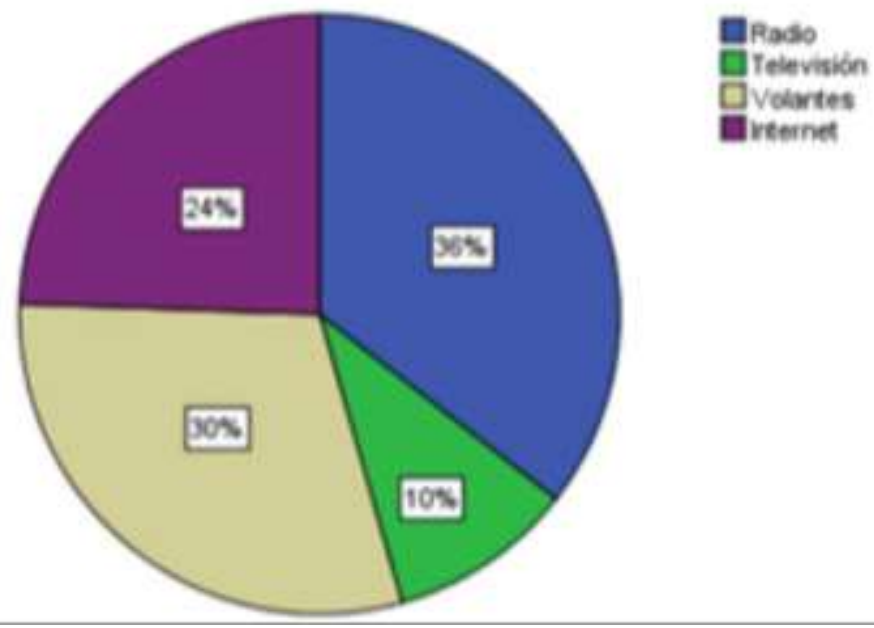

Gráfico 5. Aceptación de los medios de comunicaciones 


\section{Servicio de entrega a domicilio}

De acuerdo a los datos obtenidos el $89 \%$ dijo que sería mejor conveniente este servicio por la facilidad de los clientes al no tener tiempo, seguido del $11 \%$ que no se de este servicio por vivir cerca del negocio.

Toda empresa debe asumir una estrategia general que esté enfocada en la creación de una posición estratégica única y valiosa para la empresa (Cuadros y Samaniego, 2016).

Pasando al análisis de las estrategias que las empresas pueden adoptar para encarar tanto las fuerzas competitivas como las barreras de entrada que sugiere Porter $(1985,1987,2013)$, propone tres estrategias genéricas, que son: a) Liderazgo en el costo total, b) Diferenciación y c) Enfoque.

Como resultado, Porter (2013) sugiere que mediante la aplicación de estrategias genéricas los negocios de forma específica, las que pertenecen al sector terciario tratan de utilizar sus ventajas competitivas en los mercados o fragmentos de mercado establecidos como objetivos estratégicos. (Tabla 1)

Tabla 1. Propuesta de estrategias genéricas de Porter

\begin{tabular}{|c|c|c|c|c|}
\hline CLASIFICACIÓN & $\begin{array}{c}\text { SUB } \\
\text { CLASIFICACIÓN }\end{array}$ & DEFINICIÓN & CUANDO USAR & APLICACIÓN \\
\hline \multirow{3}{*}{$\begin{array}{l}\text { BÁSICAS DE } \\
\text { DESARROLLO(PORTER) }\end{array}$} & $\begin{array}{l}\text { LIDERAZGO EN } \\
\text { COSTOS }\end{array}$ & $\begin{array}{l}\text { Se enfoca a } \\
\text { obtener un } \\
\text { costo bajo en } \\
\text { relación a sus } \\
\text { competidores. }\end{array}$ & $\begin{array}{l}\text { Cuando una } \\
\text { empresa usa } \\
\text { correctamente } \\
\text { sus costos }\end{array}$ & $\begin{array}{l}\text { No se aplica al } \\
\text { negocio de } \\
\text { "MUNDO DE } \\
\text { ENSUEÑOS" debido } \\
\text { a la mala } \\
\text { manipulación de los } \\
\text { precios por parte de } \\
\text { la competencia. }\end{array}$ \\
\hline & DIFERENCIACIÓN & $\begin{array}{l}\text { Dar al producto } \\
\text { ventajas } \\
\text { competitivas } \\
\text { importantes } \\
\text { respecto a } \\
\text { productos } \\
\text { similares. }\end{array}$ & $\begin{array}{l}\text { Cuando el } \\
\text { producto tenga } \\
\text { características } \\
\text { importantes } \\
\text { que les haga } \\
\text { diferentes }\end{array}$ & $\begin{array}{l}\text { Se aplica al negocio } \\
\text { "MUNDO DE } \\
\text { ENSUEÑOS" que } \\
\text { ofrece productos } \\
\text { diferentes a los de } \\
\text { la competencia. }\end{array}$ \\
\hline & ENFOQUE & $\begin{array}{l}\text { Significa } \\
\text { especializarse en } \\
\text { las necesidades o } \\
\text { preferencias de } \\
\text { un grupo } \\
\text { particular de } \\
\text { compradores }\end{array}$ & $\begin{array}{l}\text { Cuando el } \\
\text { mercado meta } \\
\text { es atractivo y } \\
\text { hay } \\
\text { necesidades } \\
\text { insatisfechas } \\
\text { en cada } \\
\text { segmento. }\end{array}$ & $\begin{array}{l}\text { El negocio "MUNDO } \\
\text { DE ENSUEÑOS" no } \\
\text { se especializa en un } \\
\text { solo segmento, } \\
\text { debido a que cada } \\
\text { segmento tiene } \\
\text { necesidades } \\
\text { diferentes. }\end{array}$ \\
\hline
\end{tabular}

Fuente: Basado en (Cuadros y Samaniego, 2016). 
Las estrategias de crecimiento se hallan en la mayor parte de las estrategias empresariales y son las relacionadas al crecimiento de las ventas, participación en el mercado, beneficio o tamaño de la organización (Ruiz, 2018).

La empresa que defina un objetivo de crecimiento, la puede clasificar en tres niveles diferentes: crecimiento intensivo, crecimiento integrado y crecimiento por diversificación (Kotler, 1984; Kotler y Keller, 2012). (Tabla 2)

Tabla 2. Propuesta de estrategias de Kotler

\begin{tabular}{|c|c|c|c|c|}
\hline CLASIFICACIÓN & SUBCLASIFICACIÓN & DEFINICIÓN & CUANDO USAR & APLICACIÓN \\
\hline \multirow{3}{*}{$\begin{array}{l}\text { CRECIMIENTO } \\
\text { INTENSIVO } \\
\text { (KOTLER) }\end{array}$} & $\begin{array}{l}\text { PENETRACIÓN DE } \\
\text { MERCADO }\end{array}$ & $\begin{array}{l}\text { Consiste en } \\
\text { aumentar las } \\
\text { ventas de } \\
\text { productos } \\
\text { actuales en } \\
\text { mercados } \\
\text { actuales sin } \\
\text { alterar la oferta } \\
\text { y los } \\
\text { segmentos. }\end{array}$ & $\begin{array}{l}\text { Cuando la } \\
\text { empresa tiene un } \\
\text { buen } \\
\text { posicionamiento y } \\
\text { el mercado está } \\
\text { en crecimiento. }\end{array}$ & $\begin{array}{l}\text { El negocio "MUNDO } \\
\text { DE ENSUEÑOS" no } \\
\text { posee un buen } \\
\text { posicionamiento en } \\
\text { el mercado, y no se } \\
\text { ha expandido por lo } \\
\text { que sus ingresos no } \\
\text { se han incrementado } \\
\text { de una mejor manera } \\
\text { como la que desearía } \\
\text { el negocio. }\end{array}$ \\
\hline & $\begin{array}{l}\text { DESARROLLO DE } \\
\text { MERCADOS }\end{array}$ & $\begin{array}{l}\text { Consiste en } \\
\text { dirigirse a } \\
\text { nuevos } \\
\text { mercados con } \\
\text { los productos } \\
\text { actuales }\end{array}$ & $\begin{array}{l}\text { Cuando aumenta } \\
\text { la demanda y se } \\
\text { aprovecha la } \\
\text { experiencia de } \\
\text { mercados } \\
\text { similares }\end{array}$ & $\begin{array}{l}\text { El buscar nuevos } \\
\text { mercados } \\
\text { como la apertura de } \\
\text { un nuevo local al sur } \\
\text { de la ciudad de } \\
\text { Latacunga, es un } \\
\text { proyecto de largo } \\
\text { plazo. }\end{array}$ \\
\hline & $\begin{array}{l}\text { DESARROLLO DE } \\
\text { PRODUCTOS }\end{array}$ & $\begin{array}{l}\text { Consiste en } \\
\text { hacer } \\
\text { productos } \\
\text { nuevos o } \\
\text { mejorados para } \\
\text { mercados } \\
\text { actuales }\end{array}$ & $\begin{array}{l}\text { Cuando la } \\
\text { empresa tenga la } \\
\text { capacidad de } \\
\text { invertir en } \\
\text { productos nuevos } \\
\text { y sea una industria } \\
\text { de alta tecnología }\end{array}$ & $\begin{array}{l}\text { Debido a que el } \\
\text { negocio de "MUNDO } \\
\text { DE ENSUEÑOS" es un } \\
\text { negocio familiar y no } \\
\text { cuenta con la } \\
\text { tecnología adecuada } \\
\text { para poder llegar a } \\
\text { ser una industria; por } \\
\text { otro lado, los } \\
\text { productos que } \\
\text { comercializa son } \\
\text { provenientes del } \\
\text { Perú. }\end{array}$ \\
\hline
\end{tabular}




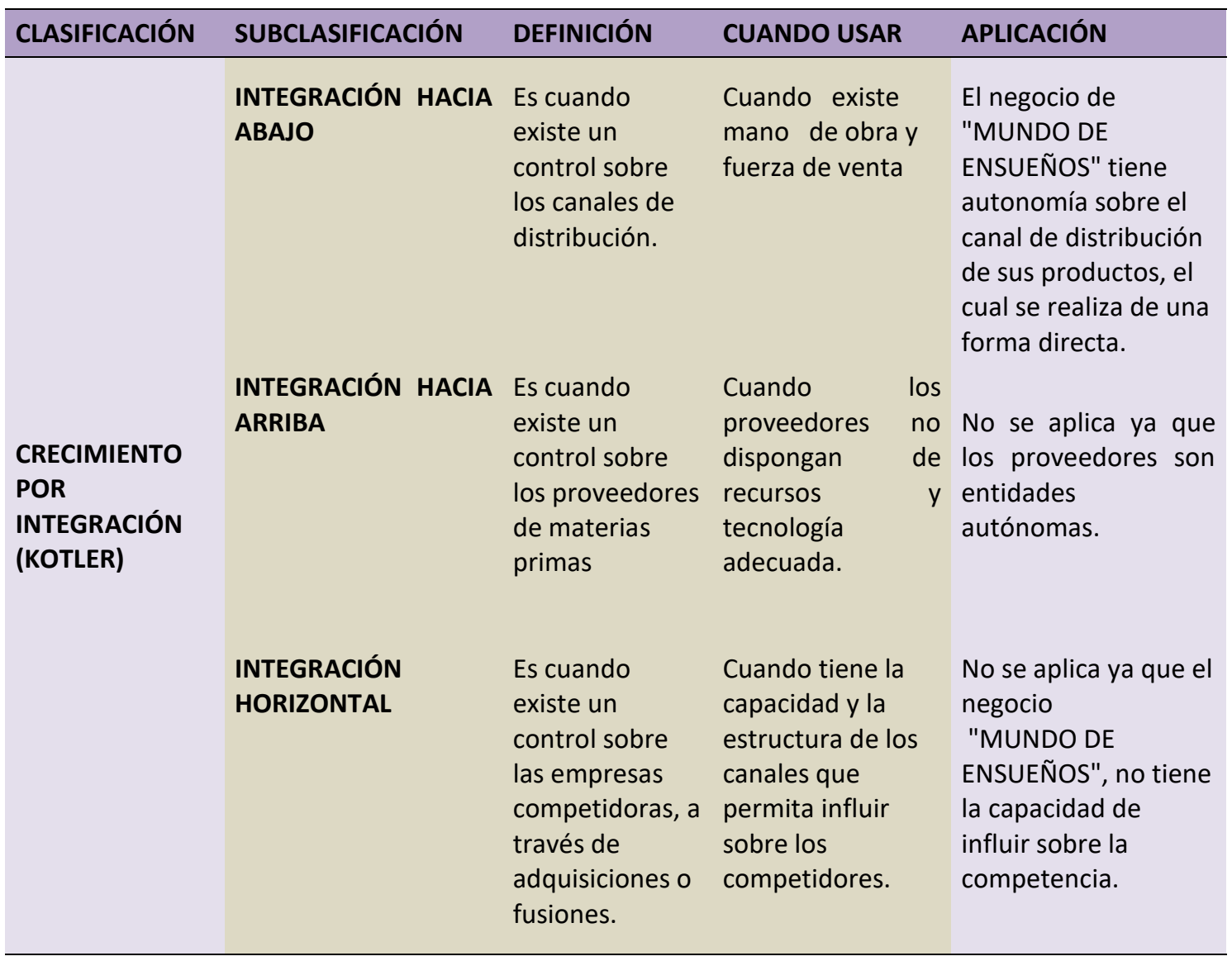

Fuente: Basado en (Kotler y Keller, 2012).

Ninguna organización puede implementar todas las estrategias, puesto que cada una sirve para situaciones específicas diferentes y cuentan con recursos limitados. La estrategia de uno o más niveles suele aplicarse en empresas grandes y diversificadas, donde posee áreas o divisiones funcionales muy bien diferenciada una de otras (David, Ramírez, Borneville, Clúa. y Márquez, 2013).

Al respecto, Peter Drucker sustenta que una empresa puede diversificarse y poseer fundamental integridad si todos los elementos mencionados se agrupan a una tecnología común (Drucker, 1987). Por otro lado, el valor acerca de dónde y cómo se alcanzarán los objetivos establecen la segunda condición de éxito empresario a través de la diversificación. Drucker propone que la unidad de mercado trabajará sobre una base diversificada sólo si se utiliza una genuina estrategia empresarial y este no será fuerte si la diversificación es un elemento agregado (Drucker, 1987). (Tabla 3). 
Tabla 3. Propuesta de estrategias de Druker

\begin{tabular}{|c|c|c|c|c|}
\hline CLASIFICACIÓN & $\begin{array}{c}\text { SUB } \\
\text { CLASIFICACIÓN }\end{array}$ & DEFINICIÓN & CUANDO USAR & APLICACIÓN \\
\hline \multirow{3}{*}{$\begin{array}{l}\text { CRECIMIENTO POR } \\
\text { DIVERSIFICACIÓN } \\
\text { (Drucker) }\end{array}$} & CONCÉNTRICA & $\begin{array}{l}\text { Nuevas } \\
\text { operaciones de } \\
\text { negocios } \\
\text { vinculadas a las } \\
\text { actividades de la } \\
\text { empresa. }\end{array}$ & $\begin{array}{l}\text { Cuando decide } \\
\text { vender } \\
\text { productos y/o } \\
\text { servicios nuevos. }\end{array}$ & $\begin{array}{l}\text { Se aplica al negocio de } \\
\text { "MUNDO DE } \\
\text { ENSUEÑOS", ya que } \\
\text { está en plena libertad } \\
\text { de poder dedicarse a } \\
\text { comercializar otro } \\
\text { tipo de productos. }\end{array}$ \\
\hline & PURA & $\begin{array}{l}\text { Nuevas áreas de } \\
\text { negocios que no } \\
\text { poseen } \\
\text { conexiones con } \\
\text { ninguna área } \\
\text { existente. }\end{array}$ & $\begin{array}{l}\text { Cuando hay } \\
\text { experiencia en el } \\
\text { mercado y no } \\
\text { hay crecimiento. }\end{array}$ & $\begin{array}{l}\text { Se aplica al negocio de } \\
\text { "MUNDO DE } \\
\text { ENSUEÑOS" ya que } \\
\text { lleva en el mercado } \\
\text { alrededor de } 4 \text { años y } \\
\text { puede ingresar a } \\
\text { comercializar otro tipo } \\
\text { de producto. }\end{array}$ \\
\hline & LIDER & $\begin{array}{l}\text { Búsqueda de } \\
\text { nuevos productos } \\
\text { y/o servicios, } \\
\text { expande } \\
\text { participación de } \\
\text { mercados. }\end{array}$ & $\begin{array}{l}\text { Cuando la } \\
\text { empresa es líder } \\
\text { en el mercado y } \\
\text { tiene una } \\
\text { participación } \\
\text { dominante. }\end{array}$ & $\begin{array}{l}\text { No se aplica al negocio } \\
\text { de "MUNDO DE } \\
\text { ENSUEÑOS" ya que el } \\
\text { distribuidor de } \\
\text { peluches es líder en el } \\
\text { sector. }\end{array}$ \\
\hline \multirow[t]{3}{*}{$\begin{array}{l}\text { COMPETITIVAS } \\
\text { (Drucker) }\end{array}$} & RETADOR & $\begin{array}{l}\text { Expandir } \\
\text { participación de } \\
\text { mercado } \\
\text { atacando al líder. }\end{array}$ & $\begin{array}{l}\text { Cuando existe la } \\
\text { capacidad y } \\
\text { recursos } \\
\text { necesarios. }\end{array}$ & $\begin{array}{l}\text { El negocio de peluches } \\
\text { "MUNDO DE } \\
\text { ENSUEÑOS" no se le } \\
\text { puede considera como } \\
\text { un retador debido a } \\
\text { que no cuenta con la } \\
\text { misma capacidad } \\
\text { financiera como el de la } \\
\text { competencia. }\end{array}$ \\
\hline & SEGUIDOR & $\begin{array}{l}\text { Clonar o imitar } \\
\text { productos y/o } \\
\text { servicios del líder. }\end{array}$ & $\begin{array}{l}\text { Cuando persigue } \\
\text { una cuota de } \\
\text { participación de } \\
\text { mercado } \\
\text { reducida y no } \\
\text { tiene tantos } \\
\text { recursos. }\end{array}$ & $\begin{array}{l}\text { El negocio de "MUNDO } \\
\text { DE ENSUEÑOS" se lo } \\
\text { puede considerar como } \\
\text { un seguidor debido a } \\
\text { que comercializa } \\
\text { productos de } \\
\text { características } \\
\text { similares. }\end{array}$ \\
\hline & ESPECIALISTA & $\begin{array}{l}\text { Empresas } \\
\text { pequeñas que } \\
\text { buscan } \\
\text { especializarse en } \\
\text { nichos de } \\
\text { mercado. }\end{array}$ & $\begin{array}{l}\text { Cuando los } \\
\text { nichos de } \\
\text { mercados son } \\
\text { duraderos y } \\
\text { rentables. }\end{array}$ & $\begin{array}{l}\text { Se aplica debido a que } \\
\text { el negocio de "MUNDO } \\
\text { DE ENSUEÑOS" busca } \\
\text { ingresar a nuevos } \\
\text { nichos de mercado } \\
\text { para mejorar su cuota } \\
\text { de participación de } \\
\text { mercado. }\end{array}$ \\
\hline
\end{tabular}

Fuente: Fuente: Basado en (David et. al., 2012). 
Indicadores de marketing (Tabla 4)

Tabla 4. Indicadores básicos para incrementar las ventas

\begin{tabular}{|c|c|c|c|}
\hline INDICADOR & FÓRMULA & INTERPRETACIÓN & META \\
\hline $\begin{array}{l}\text { Crecimiento } \\
\text { del Mercado }\end{array}$ & $\begin{array}{l}\text { vVP = Incremento delas } \\
\text { ventas propias de un } \\
\text { período a otro. } \\
\text { VTM = Ventas totales } \\
\text { del mercado o } \\
\text { segmento a nivel local. }\end{array}$ & $\begin{array}{l}\text { Permite analizar la } \\
\text { evolución del mercado } \\
\text { propio frente al mercado } \\
\text { global y revisar la cartera } \\
\text { de productos. } \\
\qquad \frac{\Delta V P}{V T M} * 100=\%\end{array}$ & $\begin{array}{l}12 \% \text { tasa de crecimiento de } \\
\text { mercado en el negocio de } \\
\text { "MUNDO DE ENSUEÑOS" }\end{array}$ \\
\hline $\begin{array}{l}\text { Participación } \\
\text { en el } \\
\text { Mercado }\end{array}$ & $\begin{array}{l}\text { VTP = Ventas totales } \\
\text { propias por servicio. } \\
\text { VTM = Ventas totales } \\
\text { del mercado. }\end{array}$ & $\begin{array}{l}\text { Permite cuantificar la } \\
\text { participación del negocio } \\
\text { en el mercado. } \\
\qquad \frac{V T P}{V T M} * 100=\%\end{array}$ & $\begin{array}{l}10 \% \text { sobre la captación de } \\
\text { mercado de la demanda } \\
\text { potencial. }\end{array}$ \\
\hline Tasa de éxito & $\begin{array}{l}\text { CC = Clientes que } \\
\text { compran el producto. } \\
\text { PC = Usuarios que usan } \\
\text { el producto. }\end{array}$ & $\begin{array}{l}\text { Evalúa el grado de } \\
\text { aceptación del producto } \\
\text { frente a la aceptación de la } \\
\text { competencia. } \\
\qquad \frac{C C}{P C} * 100=\%\end{array}$ & $\begin{array}{l}75 \% \text { del mercado de los } \\
\text { rivales de mercado. }\end{array}$ \\
\hline
\end{tabular}

CONCLUSIONES

Basado en la necesidad de detectar y analizar los factores que influían en el estancamiento de las ventas por tres años consecutivos del negocio "MUNDO DE ENSUEÑOS" se determinó que el mismo debe ingresar con una estrategia de crecimiento de mercados, lo cual significará que deberá competir con precios similares a los de la competencia. El negocio "MUNDO DE ENSUEÑOS" debe poner más énfasis en la atención al cliente utilizando estrategias propuestas para poder fidelizarlo y fomentar la lealtad a largo plazo.
Una alternativa importante para poder captar la mayor parte del mercado es mediante la capacitación en atención al cliente, técnicas de ventas y comportamiento del consumidor, lo que permitirá aumentar la fuerza de ventas.

Se aplicó indicadores de marketing que permitió saber la posición competitiva y tomar mejores decisiones gerenciales a corto, mediano y largo plazo.

Se recomienda poner en marcha las estrategias de marketing para conseguir los resultados esperados. 


\section{REFERENCIAS}

Chou, T., y Chen, F. (2004). Retail pricing strategies in recession economies: the case of Taiwan. Journal of International Marketing, 12(1), 82-102

Cuadros, C., y Samaniego, R. (2016). Estrategia de diferenciación: el caso de las empresas industriales. Ingeniería Industrial, (34), 165-174. Recuperado de http://www.redalyc.org/articulo.oa?id=3 37450992008

David, F., Ramírez, V., Borneville, E., Clúa, y Márquez, M. (2013). Conceptos de administración estratégica. México D.F.: Pearson

Drucker, P. (1987). La gerencia, Tareas, responsabilidades y prácticas. Buenos Aires. Ateneo

Farías Nazel, P. (2014). Estrategias de marketing utilizadas por las empresas chilenas para incrementar el valor de los clientes. Cuadernos de administración, 30 (51), 8-14

Fischer, Laura y Alma Navarro. 1996. Introducción a la investigación de mercados. México D.F.: McGraw-Hill

Hogan, J., Lemon, K., y Libai, B. (2004). Quantifying the ripple: word-of-mouth and advertising effectiveness. Journal of Advertising Reseach, 44 (3), 271-280

INEC. (2010). VI Censo de Población y VII de Vivienda.

Recuperado https://www.ecuadorencifras.gob.ec/est adisticas/

Kotler, P. (1984). Mercadotecnia. México D.F: Prentice Hall Hispanoamericana

Kotler, P. y Keller, K. (2012). Dirección de marketing. México D.F.: Pearson Educación

Naim, M. (2002). Venezuela Competitiva. Caracas. Editado por IESA

Porter, M. (1985). Competitive Advantage. Nueva York: First Free Press Edition. Recuperado http://94.236.206.206/dohodi.net/ books/en/Business\%20Books/Michael\%2 OPorter/Michael.Porter.Competitive.Adv antage.pdf

Porter, M. (1987). Competitive advantage to corporate strategy. Harvard Business Review, 65(3), 43-59

Porter, M. (2013). Estrategia competitiva, Editorial Continental. 42 a ed. reimpresión. México D. F: Grupo Editorial Patria

Ruiz, A. (2018). La estrategia de crecimiento intensivo de desarrollo de productos: conceptos, uso y aplicación. Researchgate. Recuperado de https://www.researchgate.net/publicatio $\mathrm{n} / 326815962$

Tamayo, M. T. (2004). El proceso de la investigación científica. (G. Noriega) México D. F.: Limusa, S 